\title{
Locally nilpotent derivations of affine domains
}

\author{
L. Makar-Limanov *
}

\begin{abstract}
Any locally nilpotent derivation of an affine domain is equivalent to a restriction of a Jacobian type derivation of a polynomial ring.
\end{abstract}

\section{Introduction.}

Let us recall that a locally nilpotent derivation is a generalization of a partial derivative of a polynomial ring. It is a derivation of a ring which when applied sufficiently many times to a given element of a ring sends it to the zero.

${ }^{*}$ The author is supported by an NSA grant. It is also a pleasure to acknowledge the hospitality of several institutions the author enjoyed while working on this project, to wit the Faculty of Mathematics and Computer Science of Nicolaus Copernicus University in Torun, Poland, the Department of Mathematics of University of Hong Kong, China, and the Max Plank Institute of Mathematics in Bonn, Germany. 
Locally nilpotent derivations (let us call them lnd) are useful though rather elusive objects. Though on "majority" of rings we do not have them at all, when we have them it is rather hard to find them and even harder to find all of them or to give some qualitative statements. Even for polynomial rings we do not know much. Of course it is rather easy to understand the situation for a polynomial ring with one variable. For two variable case it is not easy and requires a considerable effort (see [Re] or [ML2]). For three variables the kernel of a non-zero lnd is a polynomial ring in two variables ([Mi]) and there is a description of all weighted homogeneous derivations, i. e. derivations which send forms which are homogeneous relative to a given set of variables supplied with given positive weights to homogeneous forms ([Fr1], [Da2], [DR]). There are examples of lnd on polynomial rings with more than four variables with non-finitely generated kernels ([Ro], [Fr2], [DF1]) and though it is not known whether such derivations exist in the case of four generators, it is know that here there is no uniform bound on the number of generators of the kernel of an lnd ([DF2]). There are also several classes of surfaces for which we know everything as far as lnds are concerned ([ML2], [ML3], [ML4], [Da3], [Wi]) and a class of threefolds for which we know a lot ([ML1], [KML]), and that is all.

The purpose of this paper is to give a standard form for an lnd on the affine domains. This form is somewhat analogous to a matrix representation of a linear operator. Hopefully this representation of lnds will be helpful in the future research.

\section{Definitions and claim.}


Let $\mathbb{C}$ be the field of complex numbers and let $A$ be a domain over $\mathbb{C}$. A $\mathbb{C}$-linear mapping $\partial: A \rightarrow A$ is called a derivation if it distributes a product of elements of $A$ according to the Leibnitz law: $\partial(a b)=\partial(a) b+a \partial(b)$.

Constants $A^{\partial}=\{a \in A \mid \partial(a)=0\}$ of $\partial$ form a subalgebra of $A$.

A derivation is locally nilpotent if for any $a \in A$ there exists a natural $n=n(a)$ for which $\partial^{n}(a)=0$. Let us denote by $\operatorname{LND}(A)$ the set of all lnd of $A$.

Let $\mathbb{C}_{n}=\mathbb{C}\left[x_{1}, \ldots, x_{n}\right]$ be the polynomial ring with $n$ generators. A class of derivations on $\mathbb{C}_{n}$ can be obtained as follows. Take $f_{1}, \ldots, f_{n-1} \in$ $\mathbb{C}_{n}$. Define $\partial(g)=\mathrm{J}\left(f_{1}, \ldots, f_{n-1}, g\right)$ where $\mathrm{J}$ stands for the Jacobian of its arguments, that is for the determinant of the corresponding Jacobi matrix. Sometimes these derivations are called Jacobian derivations. A Jacobian derivation is also a derivation in every parameter $f_{i}$, which helps to make computations.

Now we are ready to formulate the main result.

Theorem. Let $I$ be a prime ideal of $\mathbb{C}_{n}, R$ be the factor ring $\mathbb{C}_{n} / I$, and $\pi: \mathbb{C}_{n} \rightarrow R$ be the corresponding projection. Let $\partial \in \operatorname{LND}(R)$. Then there exist elements $f_{1}, \ldots, f_{n-1} \in \mathbb{C}_{n}$ and non-zero elements $a, b \in R^{\partial}$ for which $a \partial(\pi(f))=b \pi\left(\mathrm{J}\left(f_{1}, \ldots, f_{n-1}, f\right)\right)$ for any $f \in C_{n}$.

The proof consists of eight lemmas. To make it less boring for the reader, here is an outline of what is going to happen.

Let us assume that the transcendence degree of $R$ is $n-k$. 
In the first five Lemmas we will see that it is possible to find $k$ elements $i_{1}, \ldots, i_{k} \in I$ so that $\mathrm{J}\left(i_{1}, \ldots, i_{k}, f_{k+1}, \ldots, f_{n}\right) \in I$ if and only if the elements $f_{k+1}, \ldots, f_{n}$ are algebraically dependent over $I$ (this is probably obvious to a geometer). Only in the last three Lemmas the derivation $\partial$ appears.

Exposition is made as elementary as possible to make this technique as popular as possible, possibly a misguided approach!

\section{First five Lemmas.}

Let $I$ be an ideal of $\mathbb{C}_{n}$. Choose a non-zero element $p \in I$ of the minimal possible total degree. We are not concerned, of course, with the case $p \in \mathbb{C}$. Up to renumbering of generators $x_{1}, \ldots, x_{n}$ we may assume that $p$ contains

$x_{1}$. If we consider $p$ as a polynomial in $x_{1}$ with coefficients in $\mathbb{C}\left[x_{2}, \ldots, x_{n}\right]$ then the coefficients of $p$ with positive degrees of $x_{1}$ do not belong to $I$ since their degrees are too small. By looking at all elements of $I$ as polynomials of $x_{1}$ we can find an element $p_{1} \in I$ satisfying the following two conditions:

The coefficient $q_{1}$ of the highest degree of $x_{1}$ of $p_{1}$ is not in $I$.

The degree $d$ of $p_{1}$ relative to $x_{1}$ is the smallest degree possible provided the first condition is satisfied.

The degree $d$ is positive because otherwise $p_{1} \in I$ is the coefficient of $x_{1}^{0}$ and the first condition is not satisfied.

Let $I_{1}=I \cap \mathbb{C}\left[x_{2}, \ldots, x_{n}\right]$. Find now $p_{2} \in I_{1}$ with the same property relative to $x_{2}$ (again up to a possible renumbering) and so on until we reach $k$ for which $I_{k}=0$. In the process we will obtain polynomials $p_{1}, p_{2}, \ldots, p_{k}$. 
Lemma 1. Any elements $f_{k}, \ldots, f_{n} \in \mathbb{C}_{n}$ are algebraically dependent over $I$.

Proof. We may assume that $f_{k}, \ldots, f_{n}$ are algebraically independent since otherwise the statement is clear. Let us consider the linear subspace $V_{N}$ of $\mathbb{C}_{n}$ of all polynomials of $f_{k}, \ldots, f_{n}$ with total degree relative to $f_{k}, \ldots, f_{n}$ not exceeding $N$. The linear dimension of $V_{N}$ over $\mathbb{C}$ is at least $\left(\frac{N}{n}\right)^{n-k+1}$.

Denote by deg the total degree of elements of $\mathbb{C}_{n}$ relative to $x_{1}, \ldots, x_{n}$ and by $\operatorname{deg}_{j}$ the degree relative to $x_{j}$. If $D=\max \left(\operatorname{deg}\left(f_{k}\right), \ldots, \operatorname{deg}\left(f_{n}\right)\right)$ then $\operatorname{deg}(f) \leq D N$ for any $f \in V_{N}$. Also let $e_{i}=\operatorname{deg}\left(p_{i}\right)$.

Take $p=a x_{1}^{m}+\ldots$ with $\operatorname{deg}(p) \leq D N$. Recall $p_{1}=q_{1} x_{1}^{d}+\ldots$ (Here $\ldots$ are the terms of the smaller degree in $x_{1}$.)

Let $r_{1,1}=q_{1} p-a x_{1}^{m-d} p_{1}$ if $m \geq d$. Then $\operatorname{deg}_{1}\left(r_{1,1}\right)<\operatorname{deg}_{1}(p)$. If $m<d$ take $r_{1,1}=q_{1} p$. Since $\operatorname{deg}\left(a x_{1}^{m-d}\right) \leq \operatorname{deg}(p)$ and $\operatorname{deg}\left(q_{1}\right)<\operatorname{deg}\left(p_{1}\right)$ in both cases $\operatorname{deg}\left(r_{1,1}\right) \leq D N+e_{1}$. Since $D N \geq \operatorname{deg}_{1}(p)$ after $D N$ steps like this we obtain $r_{1}$ with $\operatorname{deg}_{1}\left(r_{1}\right)<\operatorname{deg}_{1}\left(p_{1}\right)$ and $\operatorname{deg}\left(r_{1}\right) \leq D N\left(1+e_{1}\right)$.

Now apply the same procedure to $r_{1}$ and $p_{2}$. After $D N\left(1+e_{1}\right)$ steps we obtain $r_{2}$ with $\operatorname{deg}_{2}\left(r_{2}\right)<\operatorname{deg}_{2}\left(p_{2}\right)$ and $\operatorname{deg}\left(r_{2}\right) \leq D N\left(1+e_{1}\right)\left(1+e_{2}\right)$. Also $\operatorname{deg}_{1}\left(r_{2}\right) \leq \operatorname{deg}_{1}\left(r_{1}\right)$ since $\operatorname{deg}_{1}\left(q_{2}\right)=\operatorname{deg}_{1}\left(p_{2}\right)=0$.

Considering further on the pairs $r_{2}, p_{3}$, etc., we will get $r_{k}$ with $\operatorname{deg}\left(r_{k}\right) \leq$ $D N\left(1+e_{1}\right) \ldots\left(1+e_{k}\right)$ and $\operatorname{deg}_{i}\left(r_{k}\right)<\operatorname{deg}_{i}\left(p_{i}\right)$.

Take $\mathbf{m}=q_{1} q_{2}^{\left(1+e_{1}\right)} \ldots q_{k}^{\left(1+e_{1}\right) \ldots\left(1+e_{k-1}\right)}$. We observed that $\mathbf{m}^{D N} p=\sum \alpha_{i} p_{i}+$ $r_{k}$ where $\operatorname{deg}_{j}\left(r_{k}\right)<\operatorname{deg}_{j}\left(p_{j}\right)$ for $j=1, \ldots, k$ and $\operatorname{deg}\left(r_{k}\right) \leq D N(1+$ $\left.e_{1}\right) \ldots\left(1+e_{k}\right)$ if $\operatorname{deg}(p) \leq D N$. Denote $r_{k}$ by $r_{k}(p)$. It is clear that when $N$ is fixed the restriction of $p \rightarrow r_{k}(p)$ on $V_{N}$ is a linear mapping of $V_{N}$ into $\mathbb{C}_{n}$. 
Since the degrees of $r_{k}(p)$ relative to $x_{1}, \ldots, x_{k}$ are bounded and $\operatorname{deg}\left(r_{k}(p)\right) \leq$ $D N\left(1+e_{1}\right) \ldots\left(1+e_{k}\right)$, all remainders belong to a subspace $R_{N}$ of $\mathbb{C}_{n}$ with dimension not exceeding $c N^{n-k}$ where $c$ is a constant which does not depend on $N$. So $\operatorname{dim}\left(V_{N}\right)>\operatorname{dim}\left(R_{N}\right)$ for a sufficiently large $N$ and a linear mapping from $V_{N}$ to $R_{N}$ should have a kernel. It implies that there is a non-zero $f \in V_{N}$ with $r_{k}(f)=0$. So $\mathbf{m}^{D N} f=\sum \alpha_{i} p_{i} \in I$ and since $I$ is prime and all $q_{i} \notin I$ we conclude that $f \in I$.

Remark. If $f \in I$ then $r_{k}(f)=0$ for any $N$. Indeed, in this case $r=r_{k}(f) \in I$. Since $\operatorname{deg}_{1}(r)<\operatorname{deg}_{1}\left(p_{1}\right)$, from the definition of $p_{1}$ all coefficients of $r$ as a monomial in $x_{1}$ must be in $I_{1}$. Looking at these coefficients as monomials in $x_{2}$ and using $\operatorname{deg}_{2}(r)<\operatorname{deg}_{2}\left(p_{2}\right)$ and definition of $p_{2}$ we see that they in turn have all coefficients in $I_{2}$, etc.. Since $I_{k}=0$ we see that $r=0$.

Lemma 2. $\mathrm{J}\left(p_{1}, p_{2}, \ldots, p_{k}, x_{k+1}, \ldots, x_{n}\right) \notin I$.

Proof. Indeed, $\mathrm{J}\left(p_{1}, p_{2}, \ldots, p_{k}, x_{k+1}, \ldots, x_{n}\right)=\frac{\partial p_{1}}{\partial x_{1}} \frac{\partial p_{2}}{\partial x_{2}} \ldots \frac{\partial p_{k}}{\partial x_{k}}$ since only $p_{1}$ depends on $x_{1}$, only $p_{2}$ depends on $x_{2}$, etc.. But $\frac{\partial p_{i}}{\partial x_{i}} \notin I$ for all $i$ by definition of $p_{i}$, so $\frac{\partial p_{1}}{\partial x_{1}} \frac{\partial p_{2}}{\partial x_{2}} \ldots \frac{\partial p_{k}}{\partial x_{k}} \notin I$.

Lemma 3. $\mathrm{J}\left(f_{1}, \ldots, f_{k+1}, \ldots, f_{n}\right) \in I$ if $f_{1}, \ldots, f_{k+1} \in I$.

Proof. By Lemma 1 and Remark to Lemma 1 we can find a monomial M such that $\mathbf{M} f_{i}=\sum \alpha_{i, j} p_{j}$ for $i \leq k+1$. Therefore $\mathrm{J}\left(\mathbf{M} f_{1}, \ldots, \mathbf{M} f_{k+1}, f_{k+2}, \ldots, f_{n}\right)=$ 
$\mathbf{M}^{k+1} \mathrm{~J}\left(f_{1}, \ldots, f_{k+1}, \ldots, f_{n}\right)+\Delta=\sum \mathrm{J}\left(\alpha_{1, j_{1}} p_{j_{1}}, \ldots, \alpha_{k+1, j_{k+1}} p_{j_{k+1}}, \ldots, f_{n}\right)$.

Each summand $\mathrm{J}\left(\alpha_{1, j_{1}} p_{j_{1}}, \ldots, \alpha_{k+1, j_{k+1}} p_{j_{k+1}}, \ldots, f_{n}\right) \in I$ since there is only $k$ different $p_{i}$ 's. Also $\Delta \in I$ because it is a linear combination of Jacobians with coefficients containing $f_{i}$ 's where $i \leq k+1$.

So $\mathbf{M}^{k+1} \mathrm{~J}\left(f_{1}, \ldots, f_{k+1}, \ldots, f_{n}\right)=\sum \mathrm{J}\left(\alpha_{1, j_{1}} p_{j_{1}}, \ldots, \alpha_{k+1, j_{k+1}} p_{j_{k+1}}, \ldots, f_{n}\right)-$ $\Delta \in I$. Since $I$ is prime we see that $\mathrm{J}\left(f_{1}, \ldots, f_{k+1}, \ldots, f_{n}\right) \in I$.

Lemma 4. $\mathrm{J}\left(f_{1}, f_{2}, \ldots, f_{k}, f_{k+1}, \ldots, f_{n}\right) \in I$ if $f_{1}, f_{2}, \ldots, f_{k} \in I$ and $f_{k+1}, \ldots, f_{n}$ are dependent over $I$.

Proof. Let $P$ be a non-zero polynomial of $f_{k+1}, \ldots, f_{n}$ with value in $I$ of minimal total degree possible. We may assume (up to renumbering) that $P$ depends on $f_{n}$. So $\mathrm{J}\left(f_{1}, f_{2}, \ldots, f_{k}, f_{k+1}, \ldots, f_{n-1}, P\right) \in I$ by Lemma 3 . On the other hand $\mathrm{J}\left(f_{1}, f_{2}, \ldots, f_{k}, f_{k+1}, \ldots, f_{n-1}, P\right)=\frac{\partial P}{\partial f_{n}} \mathrm{~J}\left(f_{1}, f_{2}, \ldots, f_{k}, f_{k+1}, \ldots, f_{n}\right)$. Since $\frac{\partial P}{\partial f_{n}} \notin I$ it implies that $\mathrm{J}\left(f_{1}, f_{2}, \ldots, f_{k}, f_{k+1}, \ldots, f_{n}\right) \in I$.

Lemma 5. $\mathrm{J}\left(p_{1}, p_{2}, \ldots, p_{k}, f_{k+1}, \ldots, f_{n}\right) \notin I$ if $f_{k+1}, \ldots, f_{n}$ are independent over $I$.

Proof. Assume that $\mathrm{J}\left(p_{1}, p_{2}, \ldots, p_{k}, x_{k+1}, \ldots, x_{k+i}, f_{k+i+1}, \ldots, f_{n}\right) \notin I$ and that $i$ is minimal possible. From Lemma 2 we know that $i \leq n-k$. Elements $x_{k+1}, \ldots, x_{k+i}, f_{k+i+1}, \ldots, f_{n}$ are algebraically independent over $I$ by Lemma 4 . Let us consider now $x_{k+1}, \ldots, x_{k+i}, f_{k+i}, f_{k+i+1}, \ldots, f_{n}$. By Lemma 1 they are algebraically dependent over $I$. So let us take a nonzero polynomial $P$ of $x_{k+1}, \ldots, x_{k+i}, f_{k+i}, f_{k+i+1}, \ldots, f_{n}$ with value in $I$ of 
minimal total degree possible. Its degree relative to $f_{k+i}$ is positive as we noticed above. Since by assumption of the Lemma $f_{k+i}, f_{k+i+1}, \ldots, f_{n}$ are algebraically independent over $I$, polynomial $P$ (up to renumbering) depends on $x_{k+i}$.

From Lemma 3 follows that $I \ni \mathrm{J}\left(p_{1}, p_{2}, \ldots, p_{k}, x_{k+1}, \ldots, x_{k+i-1}, P, f_{k+1+i}\right.$, $\left.\ldots, f_{n}\right)=\frac{\partial P}{\partial x_{k+i}} \mathrm{~J}\left(p_{1}, p_{2}, \ldots, p_{k}, x_{k+1}, \ldots, x_{k+i}, f_{k+i+1}, \ldots, f_{n}\right)+\frac{\partial P}{\partial f_{k+i}} \mathrm{~J}\left(p_{1}, p_{2}\right.$, $\left.\ldots, p_{k}, x_{k+1}, \ldots, x_{k+i-1}, f_{k+i}, \ldots, f_{n}\right)$.

Since $\frac{\partial P}{\partial x_{k+i}} \notin I, \frac{\partial P}{\partial f_{k+i}} \notin I$, and $\mathrm{J}\left(p_{1}, p_{2}, \ldots, p_{k}, x_{k+1}, \ldots, x_{k+i}, f_{k+i+1}, \ldots, f_{n}\right) \notin$ $I$, we see that $\mathrm{J}\left(p_{1}, p_{2}, \ldots, p_{k}, x_{k+1}, \ldots, x_{k+i-1}, f_{k+i}, \ldots, f_{n}\right) \notin I$.

To avoid a contradiction we must assume that $\mathrm{J}\left(p_{1}, p_{2}, \ldots, p_{k}, x_{k+1}, \ldots, x_{k+i}\right.$, $\left.f_{k+i+1}, \ldots, f_{n}\right)$ does not contain $x_{j}$ 's at all which proves the Lemma.

\section{Additional definitions and facts about lnd.}

Let $A$ be a domain of characteristic zero, $F=\operatorname{Frac}(A)$, and $\partial \in \operatorname{LND}(A)$.

Derivation $\partial$ can be extended on $F$ by $\partial\left(a b^{-1}\right)=\partial(a) b^{-1}-a \partial(b) b^{-2}$. Denote this extension also by $\partial$.

Field $F$ contains an element $s$ for which $\partial(s)=1, s$ is transcendental over $F^{\partial}, A \subset F^{\partial}[s]$, and $F=F^{\partial}(s)$. So every element of $A$ can be looked at as a polynomial in $s$ and we can $\operatorname{define} \operatorname{deg}_{\partial}(a)$ for $a \in A$ as the degree of this polynomial. As usual, $\operatorname{deg}_{\partial}(0)=-\infty$.

Element $s=\frac{t}{\partial(t)}$ for some $t \in A$ with $\partial(t) \in A^{\partial}$. Also $F^{\partial}=\operatorname{Frac}\left(A^{\partial}\right)$ and since the transcendence degree of $F$ over $F^{\partial}$ is one we have $\operatorname{trdeg}\left(A^{\partial}\right)=$ $\operatorname{trdeg}(A)-1$ if $\operatorname{trdeg}(A)<\infty$.

All these facts can be found in [Es]. 
Two lnds of $A$ are equivalent if they determine the same degree function on $A$.

\section{Remaining Lemmas.}

Recall that $R=\mathbb{C}_{n} / I, \pi$ is the projection of $\mathbb{C}_{n}$ on $R$, and $\partial \in \operatorname{LND}(R)$. So all of the above is applicable to our setting.

We will be looking at elements of $R$ also as polynomials from $F^{\partial}[s]$ where $F$ is the field of fractions of $R, s \in F$, and $\partial(s)=1$ and denote by deg the corresponding degree.

Let $i_{1}, \ldots, i_{k} \in I$ and $f_{k+1}, \ldots, f_{n-1} \in \mathbb{C}_{n}$. From Lemma 3 it follows that $\epsilon(\pi(f))=\pi\left(\mathrm{J}\left(i_{1}, \ldots, i_{k}, f_{k+1}, \ldots, f_{n-1}, f\right)\right.$ is a well-defined derivation on $R$ since replacement of $f$ by $f+i$ in the right side does not change the left side if $i \in I$.

Let us chose now some algebraically independent $r_{k+1}, \ldots, r_{n-1} \in R^{\partial}$. It is possible since $\operatorname{trdeg}\left(R^{\partial}\right)=n-k-1$. Let $p_{k+1}, \ldots, p_{n-1} \in \mathbb{C}_{n}$ be any elements for which $\pi\left(p_{i}\right)=r_{i}$. Denote the derivation $\pi\left(\mathrm{J}\left(i_{1}, \ldots, i_{k}, p_{k+1}, \ldots, p_{n-1}, f\right)\right)$ of $R$ by $\epsilon(\mathbf{i}, \mathbf{r})(g)$ where $g=\pi(f)$. Again from Lemma 3 it is clear that $\epsilon(\mathbf{i}, \mathbf{r})(g)$ does not depend on a particular choice of $p_{k+1}, \ldots, p_{n-1}$. As always we will think about these derivations as derivations of $F$.

From Lemma 5 we know that for some choices of $\mathbf{i}$ these derivations are non-zero.

Lemma 6. Non-zero derivations $\epsilon(\mathbf{i}, \mathbf{r})$ and $\epsilon(\mathbf{i}, \mathbf{s})$ are linearly dependent over $F^{\partial}$. 
Proof. The claim is obvious if $\mathbf{r}$ and $\mathbf{s}$ are the same. Let us assume that $\epsilon(\mathbf{i}, \mathbf{r})$ and $\epsilon(\mathbf{i}, \mathbf{s})$ do not satisfy the claim and that the number of common elements of $\mathbf{r}$ and $\mathbf{s}$ is maximal possible under this condition. Up to renumbering we may assume that $s_{n-1} \notin\left\{r_{k+1}, \ldots, r_{n-1}\right\}$. Elements $r_{k+1}, \ldots, r_{n-1}, s_{n-1}$ are algebraically dependent. Let us take a non-zero polynomial $P$ of $r_{k+1}, \ldots, r_{n-1}, s_{n-1}$ with value zero of minimal total degree possible. Its degree relative to $s_{n-1}$ is positive. $P$ also must depend on at least one $r_{j} \notin\left\{s_{k+1}, \ldots, s_{n-1}\right\}$ since the elements $\left\{s_{k+1}, \ldots, s_{n-1}\right\}$ are algebraically independent. Again up to renumbering we may assume that it is $r_{n-1}$.

Let $p_{k+1}, \ldots, p_{n-1} \in \mathbb{C}_{n}$ be co-images of $r_{k+1}, \ldots, r_{n-1}$ and let $q$ be a coimage of $s_{n-1}$. So $P\left(p_{k+1}, \ldots, p_{n-1}, q\right) \in I$ and $I \ni \mathrm{J}\left(i_{1}, \ldots, i_{k}, p_{k+1}, \ldots, p_{n-2}, P, f\right)=$ $\frac{\partial P}{\partial p_{n-1}} \mathrm{~J}\left(i_{1}, \ldots, i_{k}, p_{k+1}, \ldots, p_{n-1}, f\right)+\frac{\partial P}{\partial q} \mathrm{~J}\left(i_{1}, \ldots, i_{k}, p_{k+1}, \ldots, p_{n-2}, q, f\right)$ for any $f \in \mathbb{C}_{n}$ by Lemma 3 . Therefore $\pi\left(\frac{\partial P}{\partial p_{n-1}} \mathrm{~J}\left(i_{1}, \ldots, i_{k}, p_{k+1}, \ldots, p_{n-1}, f\right)+\right.$ $\left.\frac{\partial P}{\partial q} \mathrm{~J}\left(i_{1}, \ldots, i_{k}, p_{k+1}, \ldots, p_{n-2}, q, f\right)\right)=0$. Both $\pi\left(\frac{\partial P}{\partial p_{n-1}}\right)$ and $\pi\left(\frac{\partial P}{\partial q}\right)$ are polynomials of $\partial$-constants $r_{k+1}, \ldots, r_{n-1}, s_{n-1}$, and so $\pi\left(\frac{\partial P}{\partial p_{n-1}}\right), \pi\left(\frac{\partial P}{\partial q}\right) \in R^{\partial} \backslash 0$ by the definition of $P$. So the corresponding derivations are linearly dependent over $F^{\partial}$, are both non-zero, and are linearly independent with $\epsilon(\mathbf{i}, \mathbf{s})$.

Since $\left\{s_{k+1}, \ldots, s_{n-1}\right\}$ has more common elements with $\left\{\pi\left(p_{k+1}\right), \ldots, \pi\left(p_{n-2}\right), \pi(q)\right\}$ $=\left\{r_{k+1}, \ldots, r_{n-2}, s_{n-1}\right\}$ than with $\left\{r_{k+1}, \ldots, r_{n-1}\right\}$ we reached a contradiction which proves the Lemma.

Remark. Since $\mathbf{r}$ is a transcendence basis of $R^{\partial}$, the kernel of $\epsilon(\mathbf{i}, \mathbf{r})$ is $F^{\partial}$ by Lemma 4.

This Lemma shows that the derivations $\epsilon(\mathbf{i}, \mathbf{r})$ essentially do not depend 
on $\mathbf{r}$. So let us fix a basis $\mathbf{r}$ and omit it from the notation for $\epsilon: \epsilon(\mathbf{i}, \mathbf{r})=\epsilon(\mathbf{i})$.

From Lemma 5 we know that $\epsilon(\mathbf{i})$ is a non-zero derivation for some choices of i. Recall $s \in F$ satisfying $\partial(s)=1$. If $\epsilon(\mathbf{i}) \neq 0$ then $\epsilon(\mathbf{i})(s) \neq 0$ since otherwise the kernel of $\epsilon(\mathbf{i})$ is too large. So there are choices of $i_{1}, \ldots, i_{k} \in I$ for which $q=\epsilon(\mathbf{i})(s) \neq 0$ and $\operatorname{deg}(q)$ is minimal possible. Let us fix such a $q$.

Lemma 7. For any $j_{1}, \ldots, j_{k} \in I$ the element $\epsilon(\mathbf{j})(s)$ is divisible by $q$ as an element of $F^{\partial}[s]$.

Proof. If the claim is wrong choose a pair $\mathbf{i}, \mathbf{j}$ so that $q=\epsilon(\mathbf{i})(s)$, $p=\epsilon(\mathbf{j})(s)$ is not divisible by $q$, and the number $d$ of common elements of $\mathbf{i}$ and $\mathbf{j}$ is maximal possible.

For any $h_{1}, \ldots, h_{k} \in I$ denote $\epsilon(\mathbf{h})(s)$ by $\Delta\left(h_{1}, \ldots, h_{k}\right)$. Since $\Delta\left(h_{1}, \ldots, h_{k}\right)=$ $\pi\left(\mathrm{J}\left(h_{1}, \ldots, h_{k}, p_{k+1}, \ldots, p_{n-1}, s\right)\right.$ it is skew-symmetric. Also $\mathrm{J}\left(f h_{1}, h_{2}, \ldots, h_{k}, p_{k+1}\right.$, $\left.\ldots, p_{n-1}, s\right)=h_{1} \mathrm{~J}\left(f, \ldots, h_{k}, p_{k+1}, \ldots, p_{n-1}, s\right)+f \mathrm{~J}\left(h_{1}, \ldots, h_{k}, p_{k+1}, \ldots, p_{n-1}, s\right)$ for any $f \in \mathbb{C}_{n}$. So $\pi\left(\mathrm{J}\left(f h_{1}, \ldots, h_{k}, p_{k+1}, \ldots, p_{n-1}, s\right)\right)=\pi(f) \pi\left(\mathrm{J}\left(h_{1}, \ldots, h_{k}, p_{k+1}\right.\right.$, $\left.\left.\ldots, p_{n-1}, s\right)\right)$ since $\pi\left(h_{1}\right)=0$. This can be written with a slight abuse of notations as $\Delta\left(g h_{1}, h_{2}, \ldots, h_{k}\right)=g \Delta\left(h_{1}, \ldots, h_{k}\right)$ where $g=\pi(f)$ because any co-image of $g$ in the left side gives the same right side. Since $\Delta$ is skewsymmetric $\Delta\left(h_{1}, h_{2}, \ldots, g h_{j}, \ldots, h_{k}\right)=g \Delta\left(h_{1}, \ldots, h_{k}\right)$ for any $j$.

It is easy to see now that $\delta(i)=\Delta\left(i_{1}, \ldots, i_{k-1}, i\right)$ is divisible by $q$ for any $i \in I$. Consider $q$ and $\delta(i)$ as elements of $F^{\partial}[s]$ and choose $u, v \in F^{\partial}[s]$ so that $w=u q+v \delta(i)$ is the greatest common divisor of $q$ and $\delta(i)$. If $q$ does not divide $\delta(i)$ then $\operatorname{deg}(w)<\operatorname{deg}(q)$. Since $F^{\partial}=\operatorname{Frac}\left(R^{\partial}\right)$ and 
$s=\frac{t}{\partial(t)}$ where $t \in R$ and $\partial(t) \in R^{\partial}$ we may assume that $u, v \in R$. But $\Delta\left(i_{1}, \ldots, i_{k-1}, u i_{k}+v i\right)=w$ which leads to a contradiction with the definition of $q$.

Without loss of generality we may assume that $j_{k} \notin\left\{i_{1}, \ldots, i_{k}\right\}$ and that $i_{k} \notin\left\{j_{1}, \ldots, j_{k}\right\}$. As we already know $\Delta\left(i_{1}, \ldots, i_{k-1}, j_{k}\right)=r q$ for some $r \in F^{\partial}[s]$. If $r \notin R$ find $f \in R^{\partial}$ so that $f r \in R$. For $j=f j_{k}-f r i_{k}$ we have $\Delta\left(i_{1}, \ldots, i_{k-1}, j\right)=0$. Now, $\Delta\left(j_{1}, \ldots, j_{k-1}, j+i_{k}\right)=f \Delta\left(j_{1}, \ldots, j_{k}\right)+(1-$ fr $) \Delta\left(j_{1}, \ldots, j_{k-1}, i_{k}\right)$. The element $\Delta\left(j_{1}, \ldots, j_{k-1}, i_{k}\right)$ is divisible by $q$ since the number of common elements of $\left\{i_{1}, \ldots, i_{k}\right\}$ and $\left\{j_{1}, \ldots, j_{k-1}, i_{k}\right\}$ is $d+1$. On the other hand $\Delta\left(i_{1}, \ldots, i_{k-1}, j+i_{k}\right)=q$ since $\Delta\left(i_{1}, \ldots, i_{k-1}, j\right)=0$. The sets $\left\{j_{1}, \ldots, j_{k-1}, j+i_{k}\right\}$ and $\left\{i_{1}, \ldots, i_{k-1}, j+i_{k}\right\}$ also have $d+1$ common elements, so $q$ divides $\Delta\left(j_{1}, \ldots, j_{k-1}, j+i_{k}\right)$. Therefore $f \Delta\left(j_{1}, \ldots, j_{k}\right)=$ $\Delta\left(j_{1}, \ldots, j_{k-1}, j+i_{k}\right)-(1-r) \Delta\left(j_{1}, \ldots, j_{k-1}, i_{k}\right)$ is divisible by $q$. Since $f \in R^{\partial}$ we can conclude that $\Delta\left(j_{1}, \ldots, j_{k}\right)$ is divisible by $q$ in $F^{\partial}[s]$.

Lemma 8. $q \in F^{\partial} \backslash 0$.

Proof. Let us show that $1=\pi\left(\mathrm{J}\left(x_{1}, \ldots, x_{n}\right)\right)$ is divisible by $q$ in $F^{\partial}[s]$. Write $\pi\left(x_{a}\right)=\sum f_{a, b} s^{b}$ where $f_{a, b} \in F^{\partial}$.

As we know it is possible to find a $v \in R^{\partial} \backslash 0$ so that $v \pi\left(x_{a}\right)=\sum g_{a, b} t^{b}$ where $g_{a, b} \in R^{\partial}$ and $t \in R$. We can lift these expressions into $\mathbb{C}_{n}: V x_{a}=$ $\sum G_{a, b} T^{b}+i_{a}$ where $V, G_{a, b}$, and $T$ are corresponding co-images and $i_{a} \in I$.

Let $A=\mathbb{C}_{n}\left[V^{-1}\right]$ i. e. $A$ is a subring of the field of fractions of $\mathbb{C}_{n}$ with denominators which are powers of $V$. Projection $\pi$ can be defined on $A$ and it will take it to $R\left[v^{-1}\right]$, which is a subring of $F^{\partial}[s]$. 
So $\mathrm{J}\left(x_{1}, \ldots, x_{n}\right)=\sum \mathrm{J}\left(y_{1}, \ldots, y_{n}\right)$ where $y_{a}$ is either $\frac{G_{a, b}}{V} T^{b}$ or $\frac{i_{a}}{V}$. Since $\mathrm{J}\left(\ldots, H T^{b}, \ldots\right)=T^{b} \mathrm{~J}(\ldots, H, \ldots)+b T^{b-1} H \mathrm{~J}(\ldots, T, \ldots)$ and $\mathrm{J}\left(\ldots, \frac{G}{V}, \ldots\right)=$ $V^{-1} \mathrm{~J}(\ldots, G, \ldots)-G V^{-2} \mathrm{~J}(\ldots, V, \ldots)$ we can write $\mathrm{J}\left(x_{1}, \ldots, x_{n}\right)=$ $\sum u_{m} \mathrm{~J}\left(z_{1}, \ldots, z_{n}\right)$ where $u_{m} \in \mathbb{C}\left[T, G_{1,0}, \ldots, G_{n, N}, i_{1}, \ldots, i_{n}, V^{-1}\right]$ and $z_{j}$ is either $G_{a, b}$, or $T$, or $i_{a}$.

Since $\pi\left(u_{m}\right) \in F^{\partial}[s]$ it suffices to show that $q$ divides $\pi\left(\mathrm{J}\left(z_{1}, \ldots, z_{n}\right)\right)$. If $T$ appears in this Jacobian in two positions then it is zero, so we may assume that there is at most one $T$ in it. Also by Lemma 3 at most $k$ positions have elements from $I$. If exactly $k$ positions have elements from $I$ then $q$ divides $\pi\left(\mathrm{J}\left(z_{1}, \ldots, z_{n}\right)\right)$ by Lemmas 6 and 7 . So we may assume that at least $n-k$ positions have elements $G_{a, b}$. Since $\pi\left(G_{a, b}\right) \in F^{\partial}$ these elements are algebraically dependent over $I$.

Assume that $\pi\left(\mathrm{J}\left(z_{1}, \ldots, z_{n}\right)\right)$ is not divisible by $q$ and rewrite it so that the elements from $I$ are in the beginning and $T$ is in the last position if at all. So $\mathrm{J}\left(z_{1}, \ldots, z_{n}\right)=\mathrm{J}\left(i_{1}, \ldots, i_{m}, h_{m+1}, \ldots, h_{n-1}, U\right)$ where $i_{j} \in I, \pi\left(h_{j}\right) \in F^{\partial}$, $\pi(U) \in F^{\partial} \cup t$ and $m<k$. Assume also that the number of elements from $I$ is maximal possible under this condition.

Let $P$ be a non-zero polynomial of $h_{m+1}, \ldots, h_{n-1}$ with value in $I$ and of minimal total degree possible. We may assume (up to renumbering) that $P$ depends on $h_{m+1}$. Therefore $\mathrm{J}\left(i_{1}, \ldots, i_{m}, P, h_{m+2}, \ldots, h_{n-1}, U\right)=$ $\frac{\partial P}{\partial h_{m+1}} \mathrm{~J}\left(i_{1}, \ldots, i_{m}, h_{m+1}, \ldots, h_{n-1}, U\right)$ is divisible by $q$. Since $\pi\left(\frac{\partial P}{\partial h_{m+1}}\right) \in R^{\partial} \backslash 0$ it implies that $\mathrm{J}\left(i_{1}, \ldots, i_{m}, h_{m+1}, \ldots, h_{n-1}, U\right)$ is divisible by $q$. So $q$ is a unit in $F^{\partial}[s]$, i. e. $q \in F^{\partial}$.

Find now any $i_{1}, \ldots, i_{k} \in I$ for which $\epsilon(\mathbf{i})(s)=q$ and take $\epsilon=\epsilon(\mathbf{i})$. It 
is clear that $\epsilon=\epsilon(s) \partial$. Since $q=\frac{a}{b}$ where $a, b \in R^{\partial} \backslash 0$ the Theorem is proved.

Remarks. Some forms of this Theorem appeared before. In [ML2] it was proved for any lnd of $\mathbb{C}_{n}$, and in [Da1] a more precise result was found for $n=3$. Also in [KML] a somewhat confusing form of it was proved for hypersurfaces. It should be mentioned that for hypersurfaces and complete intersections the choice of elements from the corresponding ideal is essentially unique.

Example. The following example shows that in general we can give with a Jacobian only an equivalent lnd. Let $\partial$ be an lnd on $\mathbb{C}_{4}$ given by $\partial(z)=y$, $\partial(y)=x, \partial(x)=a$, and $\partial(a)=0$. Since $\partial$ is a homogeneous operator any invariant of $\partial$ is the sum of homogeneous invariants. It is clear that there is just one invariant of degree 1 . So $J\left(f_{1}, f_{2}, f_{3}, x\right) \neq a$ if $f_{i} \in \operatorname{ker}(\partial)$. Indeed $f_{i}$ may be assumed homogeneous and then only one of them can have degree 1. Therefore $\operatorname{deg}\left(J\left(x, f_{1}, f_{2}, f_{3}\right)\right)>1$.

Acknowledgments. Many colleagues are familiar with preliminary versions of the proof and helped to improve the exposition. I want to single out Daniel Daigle and Shulim Kaliman for very helpful suggestions.

\section{References Sited}

[Da1] D. Daigle, On some properties of locally nilpotent derivations, J. Pure Appl. Algebra, 114(1997), 221-230.

[Da2] D. Daigle, On kernels of homogeneous locally nilpotent derivations of 
$k[X, Y, Z]$, Osaka J. Math. 37(2000), no. 3, 689-699.

[Da3] D. Daigle, Locally nilpotent derivations and Danielewski surfaces, Osaka J. Math. 41(2004), no. 1, 37-80.

[DF1] D. Daigle, G. Freudenburg, A counterexample to Hilbert's fourteenth problem in dimension 5, J. Algebra 221 (1999), no. 2, 528-535.

[DF2] D. Daigle, G. Freudenburg, A note on triangular derivations of $k\left[X_{1}, X_{2}, X_{3}, X_{4}\right]$, Proc. Amer. Math. Soc. 129(2001), no. 3, 657-662.

[DR] D. Daigle, P. Russell, On weighted projective planes and their affine rulings, Osaka J. Math. 38(2001), no. 1, 101-150.

[Es] A. van den Essen, Polynomial automorphisms and the Jacobian conjecture, Progress in Mathematics, 190. Birkhäuser Verlag, Basel 2000.

[Fr1] G. Freudenburg, Actions of $G_{a}$ on $\mathbb{A}^{3}$ defined by homogeneous derivations, J. Pure Appl. Algebra, 126(1998), 169-181.

[Fr2] G. Freudenburg, A counterexample to Hilbert's fourteenth problem in dimension six. Transform. Groups 5 (2000), no. 1, 61-71.

[KML] S. Kaliman, L. Makar-Limanov, On the Russell-Koras contractible threefolds, J. of Algebraic Geometry, 6(1997), 247-268 .

[ML1] L. Makar-Limanov, On the hypersurface $x+x^{2} y+z^{2}+t^{3}=0$ in $\mathbb{C}^{4}$ or a $\mathbb{C}^{3}$-like threefold which is not $\mathbb{C}^{3}$, Israel J. of Math., 96(1996), 419-429. [ML2] L. Makar-Limanov, Locally nilpotent derivations, a new ring invariant and applications, preprint.

[ML3] L. Makar-Limanov, On the group of automorphisms of a surface $x^{n} y=$ $P(z)$, Israel J. of Math., 121(2001), 113-123.

[ML4] L. Makar-Limanov, Locally nilpotent derivations on the surface $x y=$ $p(z)$, Proceedings of the Third International Algebra Conference (Tainan, 
2001), 215-219, Kluwer Acad. Publ., Dordrecht, 2003

[Mi] M. Miyanishi, Non-complete algebraic surfaces, Springer-Verlag, Berlin - New York(1981).

[Re] R. Rentschler, Operations du groupe additif sur le plane affine, C.R. Acad. Sci. Paris, 267(1968), 384-387.

[Ro] P. Roberts, An infinitely generated symbolic blow up in a power series ring and a new counterexample to Hilbert's fourteenth problem, J. Algebra 132(1990) 461-473.

[Wi] J. Wilkens, On the cancellation problem for surfaces, C.R. Acad. Sci. Paris Ser. I, 326 (1998), 1111-1116. 
Department of Mathematics \& Computer Science, Bar-Ilan University, 52900 Ramat-Gan, Israel

Department of Mathematics, Wayne State University, Detroit, MI 48202, USA

E-mail address: lml@math.wayne.edu 